\title{
Hereditary persistent distal cramps ${ }^{1}$
}

\author{
ANICA JUSIC, S. DOGAN, AND V. STOJANOVIC \\ From the Department of Neurology and Psychiatry, University of Zagreb \\ School of Medicine, Zagreb-Rebro, Yugoslavia
}

SUMMARY A disease consisting of persistent muscle cramps involving distal muscle groups that occurred in 12 members of the same family is described. The cramps appeared on exertion and in full relaxation or during sleep. In the third generation they appeared in the second decade; in the fourth and fifth generations in childhood with higher frequency and intensity of cramps. The disease is not sex linked and seems to be dominantly inherited. Electromyography showed no myotonic response on insertion. Motor unit potentials were normal. Continual waxing and waning electrical discharges corresponding to clinically visible contractions of parts of the muscles were present. Repetitive nerve stimulation caused no change in the amplitude of evoked muscle potentials. On spinal anaesthesia or nerve block the muscle contractions continued but became painless. The movements were only stopped with local infiltration of anaesthetic into the muscle. There were no cramps on ischaemic work. Drug studies revealed no benefit on carbamazepine, slight relief with meprobamate, and complete disappearance with potassium chloride. The remission outlasted the treatment for three months and then cramps of milder degree reappeared. Repeated potassium chloride treatment was not effective. The cramps increased on hydrochlorothiazide, and 12 hours after spinal anaesthesia. In the authors' opinion the disease should be considered as not belonging to any known nosological entity.

We have observed a disease appearing in many siblings of a family and in all of them with the same features. The clinical, electrophysiological, pharmacological, and laboratory analysis indi-

${ }^{1}$ Based on a paper presented at the IXth Congress of Neurological Sciences, New York, 1969. cated that the disease is of a type previously unknown.

\section{FAMILY HISTORY}

The family tree is illustrated in Fig. 1. The paternal grandmother had entirely shrunken hands; she was

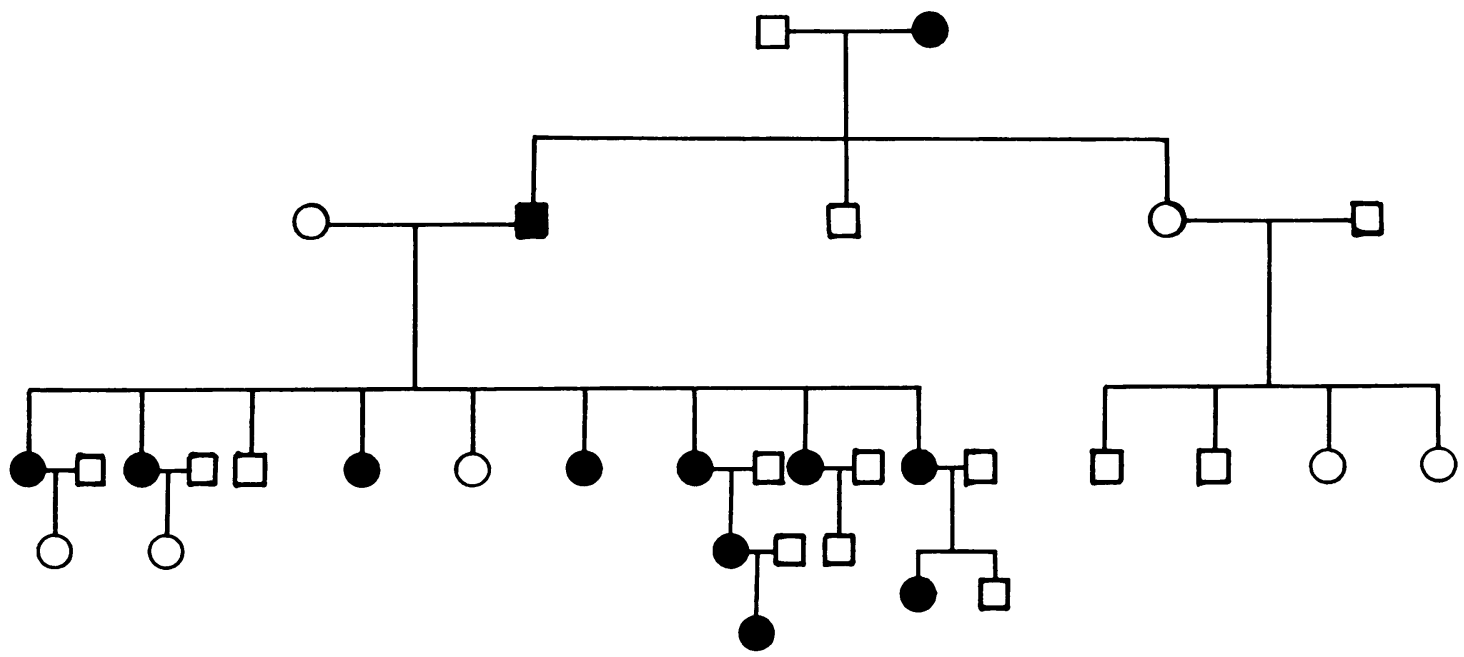

FIG. 1. Family tree. Five generations. 
unable to comb her hair. She walked with a stick, but she survived to 90 years of age. The patient's father had the first symptoms before leaving for military service. During his life painful cramps became progressively more intense but in spite of them he lived for 73 years. Until latterly he was able to walk with a stick. He had nine children. The first two, both girls, became ill at 12 and 13 years of age. The third, a boy, died when 5 years old of unknown disease. The fourth child, who was investigated most completely, became ill at the age of 13 years. She has more disabilities than any of the sibship. The fifth child was a healthy sister whose external appearance differed from the others. The next four sisters also became ill at the age of 13 years.

At the fourth generation the appearance of the illness was as follows: the first two sisters of the third generation (now 48 and 46 years old) have each a grown-up healthy daughter. The seventh sibling of the third generation, a female, has a 19 year old daughter who became ill at the age of 6 years, and a granddaughter. The latter, although only 3 years of age, already has the characteristic difficulties in the hands. The eighth sibling of the third generation has a 12 year old son with no difficulties as yet. The ninth has two children, a girl who has had her first symptoms at the age of 4 and a 5 year old healthy boy.

The father of the third generation had a brother and a sister. The sister has had four children, two girls and two boys, all of them in good health. The brother went abroad; no data about him are available.

None of the mother's family has experienced similar symptoms.

\section{CASE 1}

A 42 year old woman entered the Department of Neurology complaining of painful muscular spasms affecting especially her hands and feet.

The patient dated the onset of her difficulties at the age of 13 years. She noted symptoms whenever she was writing or wringing clothes. Over the next few years similar symptoms developed in her feet. The cramps in the feet and to a lesser extent in the hands also appeared during sleep. She had to get out of bed to press and twist her feet or hands. She could recall only a few cramps in other areas of her body. On several occasions after biting bread she was unable to open her mouth because of painful stiffness which only disappeared if she pressed down her jaw with her hands. Sometimes the painful spasms appeared in abdominal muscles when rising from a seat.

Symptoms were more severe in cold weather but also developed in hot weather after exertion. A relative change in temperature, if sudden, could influence symptoms even more.

The patient thought that her symptoms progressed slightly with age, but in spite of that she is still working at the factory. She perforated cards by pressing the pedal of the machine with a foot and changed them by hand. She used to work full time, but after some hours of repeated use of her limbs the painful stiffness in her hands and feet became almost continuous. It recovered gradually only after several hours of twisting her fingers and toes. She felt weak afterwards.

Her past medical history was unremarkable.

EXAMINATION The general physical examination revealed no abnormalities. Her blood pressure was $130 / 80 \mathrm{~mm} \mathrm{Hg}$ and heart rate 70 beats per minute and regular. Her muscles were somewhat above average size for a woman. There was no abnormal perspiration, frontal baldness, or cataracts.

Intellectually she was intact. Neurological examination disclosed abnormalities limited to a change in skeletal muscle function. Her hands were held in a more or less flexed position with fingers moving in accordance with more tonic or more jerky painful contractions of the intrinsic hand or forearm muscles. Tonic thumb opposition was especially frequent and painful.

The symptoms could be exaggerated by repeate voluntary contractions and suppressed by twisting 0 the fingers. At the peak intensity of the cramp he hands had a mottled purplish or white colour buf they never became swollen. After release of the cramp a weakness but no complete paralysis was evident There was no percussion myotonia.

The cramps of the fingers and hands were for a time almost continuous, exaggerated by exertion. They $N$ developed occasionally in the legs and were of 0 shorter duration. The trigger position was an unusual one. When she placed herself supine or-even better-prone, completely at rest, after a minute or two, different parts of the gastrocnemius muscle or $\stackrel{\unrhd}{\unrhd}$ anterior tibial compartment muscle group con- $\overrightarrow{\vec{B}}$ tracted visibly with fine and coarse twitching and rippling movements. Her feet were then flexed into the equinovarus position or moved again and again in the opposite direction with irregular jerky or tonic movements. Passive flexion of the ankle joint at that time met with a changing resistance which gradually decreased as the cramps decreased. Her calf muscles were tender to palpation. The speed and range of voluntary movements were limited during the involuntary contractions, but almost normal afterwards. Repeated voluntary contraction would not always give rise to new cramps. Cramps were $\frac{7}{0}$ almost never observed in thigh muscles. During sleep muscular contractions and feet or finger move- $N$ ments were observed many times.

The tendon jerks and abdominal reflexes were ${ }_{N}$ moderately brisk. Plantar reflexes were flexor. No disturbance of sensory function was found and sphincter functions were normal. 

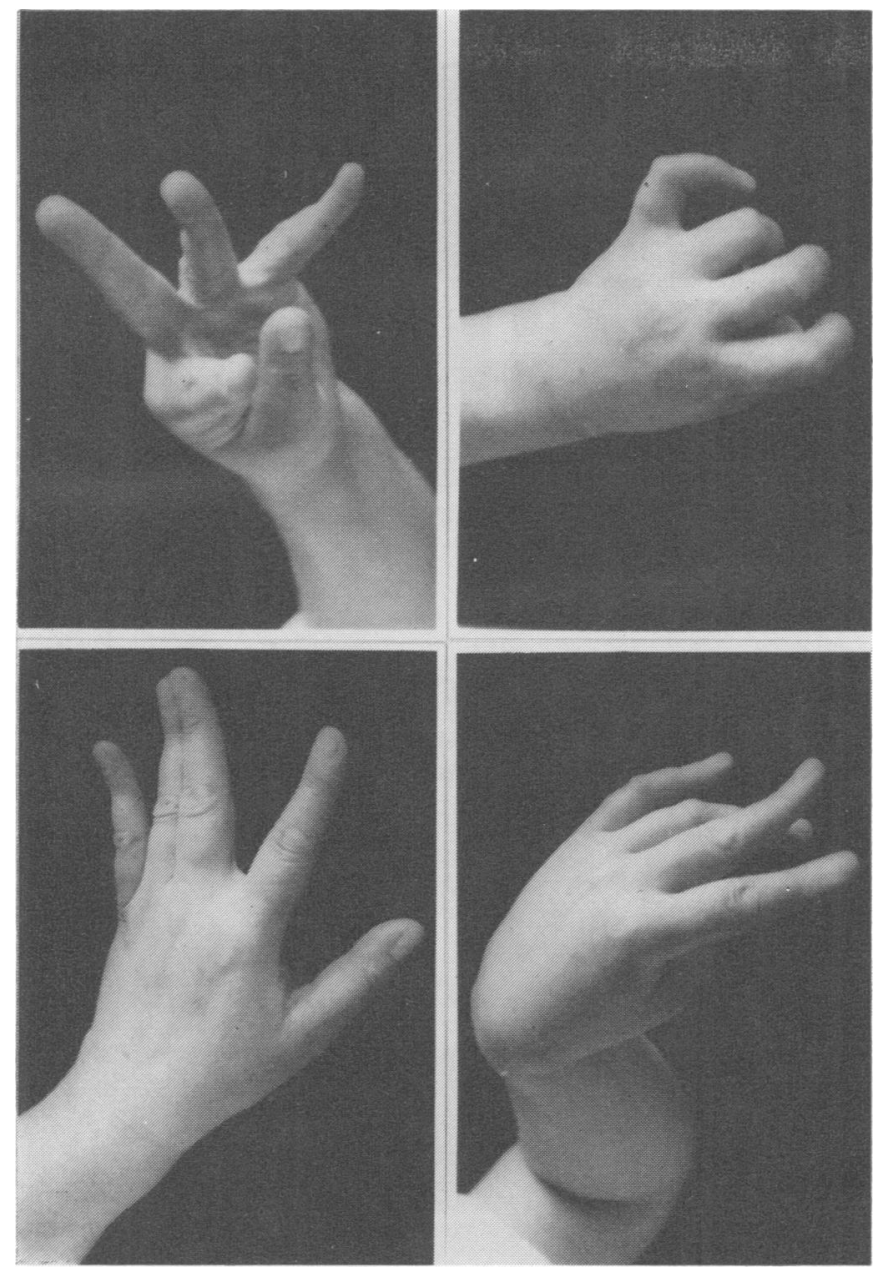

FIG. 2. The most frequent hand and finger positions caused by cramps.
LABORATORY STUDIES The complete blood count, urinalysis, blood urea nitrogen, fasting blood glucose, oral glucose tolerance, serum protein electrophoresis, immunoglobulins, serum glutamic pyruvic transaminase, serum aldolase, serum electrolyte analyses (serum calcium, phosphorus, several magnesium, sodium and potassium determinations), and cerebrospinal fluid findings were normal.

Results of radiographs of the skull, chest, and abdomen, electrocardiogram and psychological studies were all within normal limits.

SPECIAL STUdies Muscle biopsy A light microscope examination of a muscle biopsy taken from the lateral head of the gastrocnemius muscle and stained with haematoxylin-eosin was normal.

Electromyography A number of muscles of upper and lower limbs, intercostal, abdominal and paravertebral muscles and masseters were sampled with concentric needle electrodes and Tektronix dual beam 569 amplifying equipment.

No myotonic response was present on insertion or movement of the needle or on percussion of muscle. At rest continual waxing and waning electrical discharges were present with only occasional widely separated periods of electrical silence lasting several seconds. The grouped potentials corresponded to jerky finger movements and a full interference pattern was seen at the peak of the cramp. Electrical silence of longer duration developed only with clinical relief of cramps. Motor unit action potentials under voluntary control appeared normal. The interference pattern was full.

Relaxation of the antagonist muscle on voluntary contraction of agonist was full. During the cramps the activity appeared irregularly showing action potentials of variable amplitude in both muscles.

Motor and afferent nerve conduction velocities were studied using needle recording and stimulating 


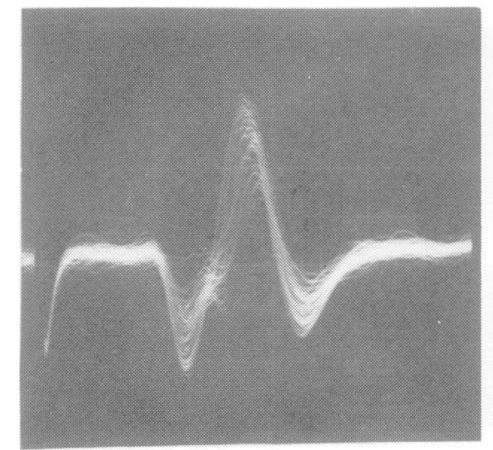

a

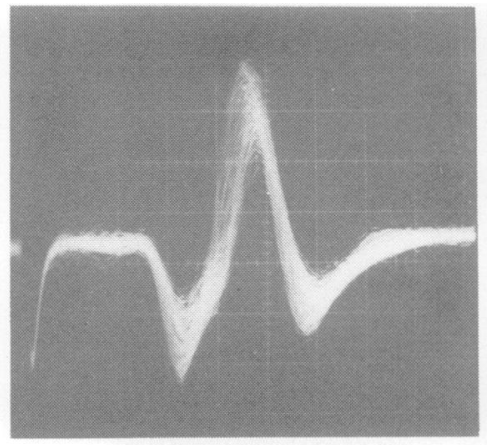

b

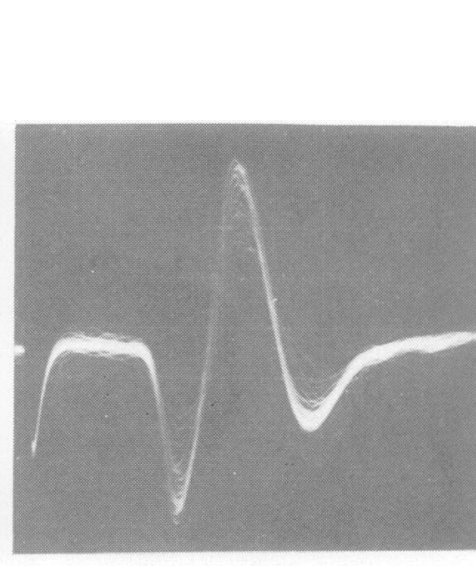

C

FIG. 3. The evoked muscle potentials in M. adductor digitorum $V$. The trains were delivered in (a) 10 cps for $\stackrel{\vec{\omega}}{\circ}$ 10 seconds, (b) 5 cps for 30 seconds, and (c) 30 cps for one second. The trains were repeated during the $20 \overrightarrow{5}^{\circ}$ minutes.

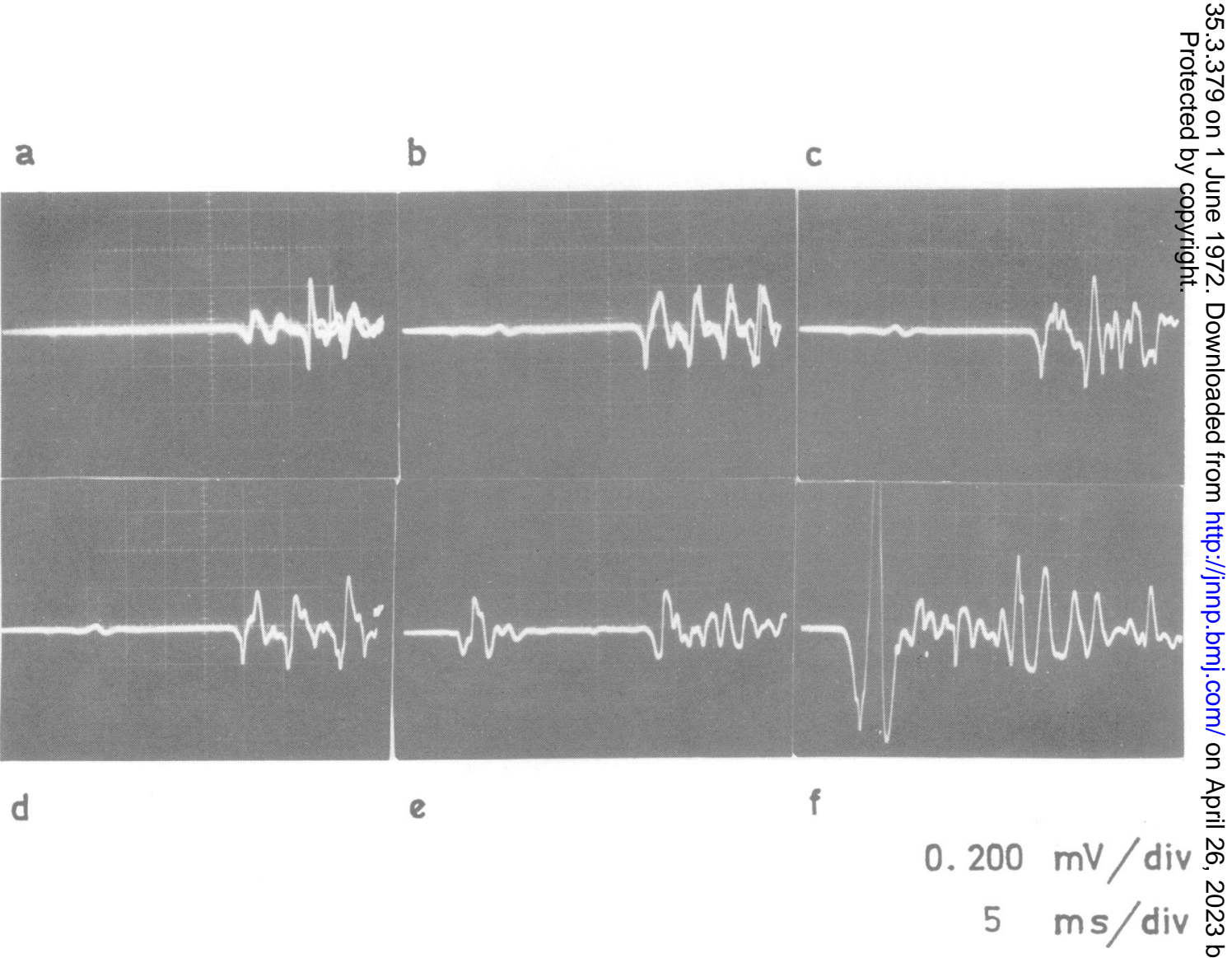

FIG. 4. The $H$ wave in gastrocnemius muscle during the cramps. Stimulation intensity in (a) $6 V$, (b) $7 V$, (c) $7 \mathrm{~V}$, (d) $7 \mathrm{~V}$, (e) $8 \mathrm{~V}$, (f) $17 \mathrm{~V}$. The $H$ wave has very long duration. 
electrodes. All the results were within the range of normal.

Repetitive nerve stimulation Trains of supramaximal electrical stimuli were delivered to the ulnar nerve at the wrist; the evoked response was recorded from the abductor digiti minimi muscle.

No significant change occurred in the amplitude of the evoked potentials. With the beginning of stimulation some cramps developed but they disappeared completely in spite of repeated applications of new trains of electrical nerve stimuli.

By stimulating the medial popliteal nerve in the popliteal fossa a typical $\mathrm{H}$ reflex could be evoked at the gastrocnemius muscle. When recorded simultaneously with the cramps it appeared highly polyphasic and with very prolonged duration (more than $20 \mathrm{msec}$ ). In the small muscles of the hands and feet the ' $F$ ' response was evoked.

Eight minutes of compression of the upper arm by a manometer cuff did not increase either the frequency or intensity of the cramps. Electromyography of the first dorsal interosseous muscle showed no multiple or triplet action potentials at rest after compression. Additional two minutes of ischaemic work brought no substantial change either.

Peripheral nerve block Spinal anaesthesia was induced with Pantocain $10 \mathrm{mg}$ injected into the subarachnoid space at the third lumbar interspace, producing complete voluntary paralysis of the legs. The involuntary muscle contractions in the calves continued with irregular jerky or tonic movements in the feet. The only difference was that the patient did not even notice the movements and there was no pain. The night afterwards the cramps became as strong as ever.

Conduction block of the ulnar and median nerves above the elbow was induced with $6 \mathrm{ml} .2 \%$ xylocain-epinephrine. The effectiveness of the block was tested by stimulating both nerves above the place of injection without producing an evoked electrical response in the muscle. All distal sensory and voluntary motor functions of ulnar and median nerves were absent afterwards. Nevertheless, involuntary tonic or jerky movements of the fingers persisted. The patient noticed them only by looking at them. The movements were now painless.

Local infiltration of the thenar eminence with $2 \mathrm{ml} .2 \%$ xylocain was the only way to stop the thumb movements for more than two hours.

DRUG STUDIES 1. Carbamazepine, prescribed in doses of $200 \mathrm{mg}$ orally three times daily, had no influence on her symptoms.

2. Diazepam, $5 \mathrm{mg}$ orally three times daily, had no effect.

3. Meprobamate, prescribed in doses of $400 \mathrm{mg}$ orally three times daily, made the nocturnal cramps less troublesome. For the first time after 20 years she slept the whole night. But the favourable influence did not last for long. Ten days later the cramps became more intense, and sleep was disturbed again, although to a lesser degree than before administration of the drug. Results were not improved by increased dosage.

4. Corticotropin (ACTH), 100 i.u. daily for two weeks did not influence the symptoms.

5. Hydrochlorothiazide, prescribed in addition to ACTH therapy, $25 \mathrm{mg}$ orally twice daily, made symptoms worse even by the second day of administration, almost as bad as the day after the spinal anaesthesia.

6. Neostigmine, $1.5 \mathrm{mg}$ intramuscularly had no effect.

7. Biperidol, three times daily $5 \mathrm{mg}$ intramuscularly had no effect.

8. Potassium chloride, $2.0 \mathrm{mg}$ daily, resulted in two days with substantial reduction and finally in total disappearance of cramps for the first time during her illness. Some change for the better was already obvious on the first day of potassium chloride therapy. After 10 days the therapy was discontinued but the condition continued to improve and four weeks later there were almost no symptoms. She was even able to wash her clothes with almost no cramps. It was three months later before the cramps reappeared. This time potassium intake had no substantial influence on the symptoms.

\section{CASES 2, 3, AND 4}

These cases are the first, the second, and the youngest sibling of the case 1 sibship. Their signs and symptoms were the same as those described in case 1 . The difference was only one of degree. They were clinically examined and electromyography was carried out in one.

\section{DISCUSSION}

The syndrome described has to be clearly differentiated from others which have some features or some superficial resemblances in common.

It is readily differentiated from myotonia of muscle. Although the patients described sometimes find difficulty in relaxing their intrinsic hand muscles completely, their cramps were worse by repeated motions. Their cramps also appear during full relaxation after some hours of sleep (we are not sure if they have some correlation with rapid eye movement sleep), which never occurs with myotonia. EMG findings in myotonia are quite different, with repetitive potentials after percussion of the muscle or on movement of the recording needle. 
The symptoms of this family are made worse by cold or a sudden change in temperature, but they are present also at normal temperature or in hot weather. After a period of strong cramps some weakness or tiredness may be observed but not a paralysis. Neostigmine did not aggravate cramps as it did in paramyotonia (Magee, 1966).

In McArdle's disease, or muscle phosphorylase deficiency, the clinical hallmarks (Rowland, Lovelace, Schotland, Araki, and Carmel, 1967) are muscle cramps and exercise intolerance, contracture after ischaemic work, and episodic myoglobinuria. Our patients never observed a dark urine. Two minutes of ischaemic work induced no cramps. The cramps developed during full relaxation in bed and during sleep too. Electromyography at the maximum of the cramps showed an interference pattern. After supramaximal nerve stimulation there was no decrement in the evoked muscle potential (Dyken, Smith, and Peake, 1967).

Diagnostic criteria of the 'stiff-man' syndrome include generalized muscular stiffness which disappears during sleep, a normal neurological examination apart from the muscle stiffness, continual EMG activity at rest, depression of EMG activity, and muscle stiffness with spinal anaesthesia and peripheral nerve blocks. The patient improves with treatment by carbamazepine.

The cases described displayed more or less transient painful stiffness of the intrinsic hand muscles. Symptoms of a lesser degree could be found in the forearm or calf muscles. Their appearance in the trunk or at masticatory muscles was very rare indeed. The painful contractions regularly roused the patient from sleep. The rest of the neurological examination was normal in both conditions. EMG activity was discontinuous in our cases corresponding to clinically visible contractions. There was no activity at rest. Neither the spinal anaesthesia nor peripheral nerve blocks stopped the appearance of contractions. They only made them painless. The carbamazepine therapy had no influence. The symptoms improved, for some months only, on potassium administration.

In basal ganglia disorders like athetosis, the slow involuntary movements of hands and feet $\vec{F}$ are not painful and they disappear during sleep, spinal or conduction anaesthesia.

The cramps in peripheral nervous system lesions like polyneuropathy or in amyotrophic lateral sclerosis are accompanied by motor, sensory, and reflex signs. The electromyographic findings are those of a neurogenic lesion.

Cramps in healthy adults appear irregularly only from time to time in calves or in small foot muscles. They appear with movements or during sleep and disappear on stretching or twisting the muscles. They differ from those of the family described quantitatively and in distribution.

The authors are indebted to Professor E. Kugelberg Neurologiska kliniken, Karolinska sjukhuset, Stocke holm, for differential-diagnostic suggestions. The $\$$ are grateful to Mrs. Fronjek Nada for skilfư assistance in electrophysiological studies.

\section{REFERENCES}

Dyken, M. L., Smith, D. M., and Peake, R. L. (1967). An electromyographic diagnostic screening test in McArdle's disease and a case report. Neurology (Minneap.), 17, 45-50.

Magee, K. R. (1966). Paramyotonia congenita. Archives of Neurology, 14, 590-594.

Rowland, L. P., Lovelace, R. E., Schotland, D. L., Araki, S., and Carmel, P. (1967). The clinical diagnosis of McArdle's disease. Neurology (Minneap.), 17, 93-100. 